ANNALES

POLONICI MATHEMATICI

LVII.3 (1992)

\title{
A method of construction of an invariant measure
}

by ANToni Leon Dawidowicz (Kraków)

Abstract. A method of construction of an invariant measure on a function space is presented.

0 . Introduction. The problem of existence of a measure invariant with respect to the dynamical system generated by the differential equation

$$
\frac{\partial u}{\partial t}+c(x) \frac{\partial u}{\partial x}=f(u)
$$

has been considered by Lasota [4], Rudnicki [5] and the author [1], [2]. At present, there are various theorems on the existence and properties of such measures. In this paper we present a general method of construction of an invariant measure.

1. Formulation of the result. Consider the differential equation

$$
\frac{\partial u}{\partial t}+x \frac{\partial u}{\partial x}=\lambda u, \quad t \geq 0,0 \leq x \leq 1,
$$

with the initial condition

$$
u(0 ; x)=v(x)
$$

and the boundary condition

$$
u(t ; 0)=0 .
$$

This problem generates a semidynamical system on the space $V$ of all Lipschitz functions on the interval $[0 ; 1]$ vanishing at 0 by the formula

$$
T_{t} v(x)=e^{\lambda t} v\left(x e^{-t}\right)
$$

Denote by $\left\|v^{\prime}\right\|$ the maximal Lipschitz constant of $v$, i.e.

$$
\left\|v^{\prime}\right\|=\sup _{0 \leq x<y \leq 1} \frac{|v(y)-v(x)|}{y-x} .
$$

1991 Mathematics Subject Classification: Primary 28D05.

Key words and phrases: dynamical system, invariant measure. 
For Lipschitz functions this supremum is finite.

Definition. Let $\lambda>1$. A measure $\mu$ on $V$ satisfies assumption $\mathrm{A}$ if there exists a sequence $\left\{\varrho_{n}\right\}$ of positive real numbers and a positive constant $K$ such that

$$
\begin{gathered}
\frac{\varrho_{n}}{2^{(\lambda-1) n}} \leq K, \quad n=1,2, \ldots, \\
\sum_{n=0}^{\infty} \mu\left(\left\{v:\left\|v^{\prime}\right\| \geq \varrho_{n}\right\}\right)<\infty .
\end{gathered}
$$

THEOREM 1. Let $\lambda>1$ and suppose $\mu$ satisfies assumption $\mathrm{A}$. Then we can construct a $T_{1}$-invariant measure $\widehat{\mu}$. The construction has the following property: If $\mu$ is $T_{1}$-invariant, then $\widehat{\mu}=\mu$.

2. Proof of Theorem 1. Let $A: V \rightarrow V$ be given by $(A v)(x)=$ $v\left(\frac{1}{2}(x+1)\right)-v\left(\frac{1}{2}\right)$ and let $\mu^{\prime}(E)=\mu\left(A^{-1}(E)\right)$. Clearly $\mu^{\prime}$ also satisfies (i), (ii) (with another sequence $\left\{\varrho_{n}\right\}$ ). Define $\Phi: V^{\mathbb{N}} \rightarrow \mathcal{P}(V)$ by

$$
\begin{array}{r}
w \in \Phi\left(v_{0}, v_{1}, \ldots\right) \Leftrightarrow \forall n, w\left(2^{-n-1}(x+1)\right)-w\left(2^{-n-1}\right)=2^{-\lambda n} v_{n}(x) \\
\text { for } 0 \leq x \leq 1 .
\end{array}
$$

This condition determines the values of $w$ on the interval $\left[2^{-n-1} ; 2^{-n}\right]$ up to an additive constant. Since $w(0)=0$ the condition $w \in \Phi\left(v_{0}, v_{1}, \ldots\right)$ can be satisfied by at most one function $w$. Hence card $\Phi\left(v_{0}, v_{1}, \ldots\right) \leq 1$.

Let $\widehat{\mu}^{\prime}$ be the product measure on $V^{\mathbb{N}}$. We claim that $\widehat{\mu}^{\prime}\left(\left\{\left(v_{0}, v_{1}, \ldots\right)\right.\right.$ : $\left.\left.\Phi\left(v_{0}, v_{1}, \ldots\right)=\emptyset\right\}\right)=0$. First suppose the sequence $\left\{v_{n}\right\}$ satisfies

$$
\exists n_{0} \forall n \geq n_{0} \quad\left\|v_{n}^{\prime}\right\| \leq \varrho_{n} .
$$

Let $\bar{w}(x)=2^{-\lambda n} v_{n}\left(2^{n+1} x-1\right)+C_{n}$ for $x \in\left[2^{-n-1} ; 2^{-n}\right]$ where the constant $C_{0}$ is arbitrary and $\left\{C_{n}\right\}$ is a sequence such that $\bar{w}$ is continuous on $(0 ; 1]$. Since, for $n \geq n_{0}, \bar{w} \mid\left[2^{-n-1} ; 2^{-n}\right]$ satisfies the Lipschitz condition with constant $2 \varrho_{n} 2^{-(\lambda-1) n} \leq 2 K$, the function $\bar{w}$ also satisfies the Lipschitz condition and in consequence $\lim _{x \rightarrow 0} \bar{w}(x)$ exists. Define

$$
w(x)=\bar{w}(x)-\lim _{y \rightarrow 0} \bar{w}(y)
$$

for $x>0$ and $w(0)=0$. Hence $w \in \Phi\left(v_{0}, v_{1}, \ldots\right)$ and in consequence for every sequence $\left(v_{0}, v_{1}, \ldots\right)$ satisfying $(7), \Phi\left(v_{0}, v_{1}, \ldots\right)$ is nonempty. Moreover, from the Borel-Cantelli lemma it follows that the set of sequences satisfying condition (7) has full measure.

Now, let $\widehat{\mu}$ be defined by

$$
\widehat{\mu}(E)=\widehat{\mu}^{\prime}\left(\left\{\left(v_{0}, v_{1}, \ldots\right): \Phi\left(v_{0}, v_{1}, \ldots\right) \subset E\right\}\right) .
$$

Clearly, $\widehat{\mu}$ is the transport of $\widehat{\mu}^{\prime}$ by a map defined on a full-measure set. The invariance and the ergodicity follow from an argument analogous to that 
in [1]. From the construction it also follows that if $\mu$ is $T_{1}$-invariant, then $\mu=\hat{\mu}$.

Remark. By the same method as in [1] a $T$-invariant measure can be constructed.

\section{Properties of the measure $\widehat{\mu}$}

THEOREM 2. The measure $\hat{\mu}$ is defined on the $\sigma$-algebra of Borel sets for the topology of uniform convergence.

Proof. Let $\Sigma$ be the $\sigma$-algebra on which $\widehat{\mu}$ is defined, i.e. $E \in \Sigma$ if and only if $(\tau \Phi)^{-1}(E)$ is $\widehat{\mu}^{\prime}$-measurable where $\tau \Phi\left(v_{0}, v_{1}, \ldots\right)$ denotes the unique element of $\Phi\left(v_{0}, v_{1}, \ldots\right)$. Since $V$ is a separable space it is sufficient to prove that if $\widetilde{v} \in V, \varepsilon>0$ then $U(\widetilde{v} ; \varepsilon)=\left\{v: \sup _{x \in[0 ; 1]}|v(x)-\widetilde{v}(x)|<\varepsilon\right\}$. In [2] (Lemma 4) it is proved that the map

$$
\left(v_{0}, v_{1}, \ldots\right) \mapsto \sup _{x \in[0 ; 1]}\left|\tau \Phi\left(v_{0}, v_{1}, \ldots\right)(x)-\widetilde{v}(x)\right|
$$

is measurable, which completes the proof.

THEOREM 3. If $\mu$ is positive on open nonempty sets, then so is $\tilde{\mu}$.

Proof. First, let $G(n ; \varepsilon)=\left\{v \in V: \forall x \in\left[0 ; 2^{-n}\right],|v(x)|<\varepsilon\right\}$. From the proof of Lemma 5 of [2] it follows that if $\widehat{\mu}\left(G\left(n ; 2^{\lambda} \varepsilon\right)\right)>0$, then there exists $\varepsilon^{\prime}<\varepsilon$ such that $\widehat{\mu}\left(G\left(n+1 ; \varepsilon^{\prime}\right)\right)>0$. Clearly $\widehat{\mu}(G(n ; \varepsilon))=$ $\widehat{\mu}\left(G\left(n+1 ; \varepsilon^{\prime}\right)\right) \hat{\mu}^{\prime}\left(\left\{v \in V:\|v\|<2^{\lambda n}\left(\varepsilon-\varepsilon^{\prime}\right)\right\}\right)>0$. By an argument analogous to that in [2] it follows that $\widehat{\mu}(G(n ; \varepsilon))>0$. The end of proof is also analogous to [2].

\section{Examples}

EXAMPLE 1. Let $\left\{\sigma_{n}\right\}$ and $\left\{p_{n}\right\}$ be as in [1] and let $\mu(E)=\sum_{\sigma_{n} \in E} p_{n}$. In this situation the measure obtained from $\mu$ by the procedure presented in Theorem 1 is the measure considered in [1], [2] (clearly, with another sequence $\left\{\sigma_{n}\right\}$ ).

Example 2. Let $\mu_{W}$ be the Wiener measure on $C[0 ; 1]$ and let $I$ : $C[0 ; 1] \rightarrow V$ be defined by the formula

$$
(I v)(x)=\int_{0}^{x} v(s) d s
$$

Let $\mu$ be the transport of the Wiener measure by $I$. Using [4] it can be proved that $\mu$ satisfies assumption $\mathrm{A}$. By the presented procedure we can obtain a Gaussian $T_{1}$-invariant measure. 


\section{References}

[1] A. L. Dawidowicz, On the existence of an invariant measure for the dynamical system generated by partial differential equation, Ann. Polon. Math. 41 (1983), 129137.

[2] -, On the positivity of an invariant measure on open non-empty sets, ibid. 50 (1989), 185-190.

[3] T. Hida, Brownian Motion, Springer, New York 1980.

[4] A. Lasota, Invariant measures and a linear model of turbulence, Rend. Sem. Mat. Univ. Padova 61 (1979), 39-48.

[5] R. Rudnicki, Invariant measure for the flow of a first order partial differential equation, Ergodic Theory Dynamical Systems 5 (1985), 437-446.

INSTITUTE OF MATHEMATICS

JAGIELLONIAN UNIVERSITY

REYMONTA 4

30-059 KRAKOW, POLAND

Reçu par la Rédaction le 10.8.1989

Révisé le 10.3.1992 